\title{
DFT Models of Molecular Species in Carbonate Molten Salts
}

\author{
W. Robert Carper, ${ }^{* \dagger}$ Phillip G. Wahlbeck, ${ }^{\dagger}$ and Trevor R. Griffiths ${ }^{\ddagger}$ \\ ${ }^{\dagger}$ Department of Chemistry, Wichita State University, Wichita, Kansas 67260-0051, United States \\ ${ }^{\ddagger}$ Redston Trevor Consulting Ltd., 58 High Ash Avenue, Leeds LS17 8RF, U.K.
}

ABSTRACT: Raman spectra of high temperature carbonate melts are correlated with carbonate species modeled at $923 \mathrm{~K}$ using B3LYP/ $(6-311+\mathrm{G}(2 \mathrm{~d}, \mathrm{p}))$ density functional

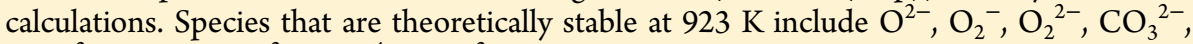
$\mathrm{C}_{2} \mathrm{O}_{6}{ }^{2-}, \mathrm{CO}_{4}^{-}, \mathrm{CO}_{4}{ }^{2-}, \mathrm{CO}_{4}{ }^{4-}, \mathrm{CO}_{5}{ }^{2-}, \mathrm{KCO}_{4}^{-}, \mathrm{LiCO}_{4}{ }^{-}, \mathrm{KO}_{2}^{-}, \mathrm{LiO}_{2}^{-}, \mathrm{NaO}_{2}^{-}, \mathrm{KO}_{2}, \mathrm{LiO}_{2}$, $\mathrm{NaO}_{2}, \mathrm{KCO}_{3}{ }^{-}, \mathrm{LiCO}_{3}{ }^{-}$, and $\mathrm{NaCO}_{3}{ }^{-}$. Triangular, linear, and bent forms are theoretically possible for $\mathrm{KO}_{2}{ }^{-}$and $\mathrm{NaO}_{2}{ }^{-}$. Triangular and linear forms may exist for $\mathrm{LiO}_{2}^{-}$. Linear and triangular versions are theoretically possible for $\mathrm{LiO}_{2}{ }^{-}$and $\mathrm{KO}_{2}$. A triangular version of $\mathrm{NaO}_{2}$ may exist. The correlation between measured and theoretical Raman spectra indicate that monovalent cations are to be included in several of the species that produce Raman spectra.

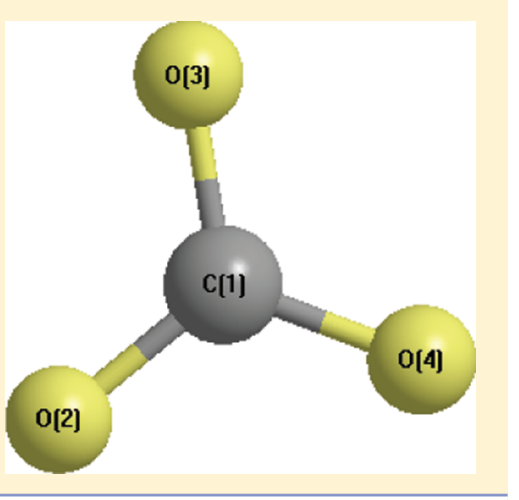

\section{INTRODUCTION}

Molten carbonate research plays an important role in several areas that include the development of efficient fuel cells, ${ }^{1-10}$ spent nuclear fuel degradation, ${ }^{11,12}$ and nerve gas oxidation. ${ }^{13}$ Research developments in these seemingly unrelated areas are dependent on the correct identification of various carbonate related species that exist in carbonate melts.

In particular, molten carbonate fuel cell (MCFC) technology is a rapidly growing area of energy research. Molten carbonate fuel cells have the ability to reach fuel conversion to electricity efficiencies of $50 \%$ and thermal efficiencies of greater than $80 \%{ }^{7-10}$ As molten carbonate fuel cells are being developed, the identification of active species in molten carbonates becomes critical. Raman spectroscopy has been used in recent years to identify possible species found in molten carbonates. ${ }^{1-6}$ The advantage of using Raman spectra rather than IR spectra is due to the complexity of overlapping bands in IR spectra that are difficult to deconvolute. Assignments of Raman absorption bands have been made by a number of investigators. ${ }^{1-6}$ Although these assignments appear to be reasonable, they lack detailed theoretical support of the type that has proved useful in similar studies of ionic liquids. ${ }^{14-16}$ In recent years, investigators have used $a b$ initio calculations and Raman spectra to probe both the overall and local structures of ionic liquids as outlined in detail in several excellent reviews. ${ }^{17,18}$

In this study, a number of theoretically stable oxygencontaining species including carbonates have been modeled. The calculated Raman spectra are seen to match the reported Raman spectra with a high degree of accuracy. Previously ignored carbonate and oxygen-containing species are identified as major contributors to the Raman spectral bands.

\section{METHODS}

Computational Methods. The density functional theory (DFT) calculations were obtained using Gaussian 03 in a tight fit configuration. ${ }^{19}$ The B3LYP $(6-311+(2 \mathrm{~d}, \mathrm{p}))$ level of computation was used for all structures. Calculated vibrational frequencies of the various structures contain no imaginary frequencies, ensuring the presence of a minimum. The eigenvectors for each normal mode were displayed on the computer and the normal modes were assigned to specific group vibrations in a manner similar to previous vibrational frequency studies of ionic liquids. $^{14-16}$

Frequency Correlations. The correlations between calculated and experimental spectra are based primarily on Raman peak intensities and frequencies $\left(\mathrm{cm}^{-1}\right) .{ }^{14-16}$ This approach avoids the IR problem in cases where adjacent experimental frequencies overlap and require deconvolution.

Molecular Structures. The calculated gas phase structures include the following: $\mathrm{O}^{2-}, \mathrm{O}_{2}^{-}, \mathrm{O}_{2}{ }^{2-}, \mathrm{CO}_{3}{ }^{2-}, \mathrm{C}_{2} \mathrm{O}_{6}{ }^{2-}, \mathrm{CO}_{4}{ }^{-}$, $\mathrm{CO}_{4}{ }^{2-}, \mathrm{CO}_{4}{ }^{4-}, \mathrm{CO}_{5}{ }^{2-}, \mathrm{KCO}_{4}{ }^{-}, \mathrm{LiCO}_{4}{ }^{-}, \mathrm{KO}_{2}{ }^{-}$(three versions), $\mathrm{LiO}_{2}^{-}$(two versions), $\mathrm{NaO}_{2}^{-}$(three versions), $\mathrm{KO}_{2}$ (two versions), $\mathrm{LiO}_{2}$ (two versions), $\mathrm{NaO}_{2}, \mathrm{KCO}_{3}{ }^{-}, \mathrm{LiCO}_{3}{ }^{-}$, and $\mathrm{NaCO}_{3}{ }^{-}$. These and other related structures were determined at the $\mathrm{B} 3 \mathrm{LYP} /(6-311+\mathrm{G}(2 \mathrm{~d}, \mathrm{p}))$ level of computation. DFT theory is usually quite successful in predicting accurate molecular structure and vibrational frequencies. ${ }^{17,18}$

\section{RESULTS AND DISCUSSION}

Gas Phase Structures. Figures 1-25 contain the DFT structures determined at the B3LYP/ $(6-311+\mathrm{G}(\mathrm{d}, \mathrm{p}))$ level of computation. The linear structures of $\mathrm{CO}_{2}, \mathrm{O}_{2}, \mathrm{O}_{2}^{-}$, and $\mathrm{O}_{2}{ }^{2-}$ are not shown for the sake of brevity.

The possible existence of a number of these species is somewhat unexpected. In addition to the usual planar form of $\mathrm{CO}_{3}{ }^{2-}$ (Figure 1), a stable planar form of $\mathrm{CO}_{3}{ }^{-}$(Figure 2) may

Received: February 20, 2012

Revised: April 11, 2012

Published: April 18, 2012 


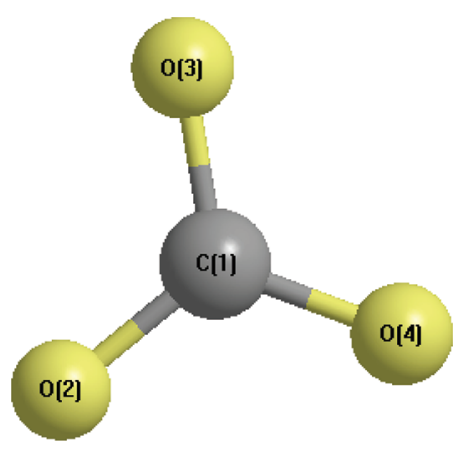

Figure 1. Planar $\mathrm{CO}_{3}^{2-}$. All $\mathrm{C}-\mathrm{O}$ distances $=1.306 \AA$.

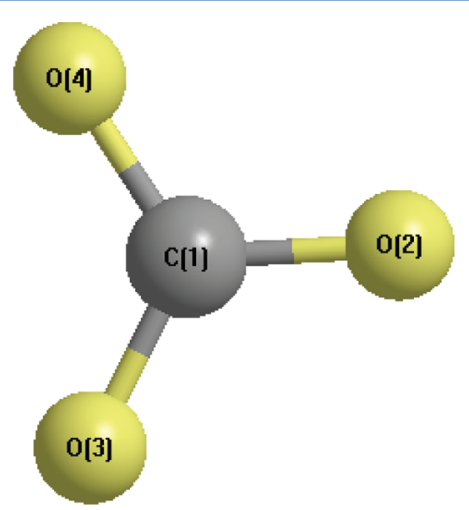

Figure 2. Planar $\mathrm{CO}_{3}^{-}$. All $\mathrm{C}-\mathrm{O}$ distances $=1.270 \AA$.

also exist. Tetrahedral structures of $\mathrm{CO}_{4}{ }^{-}, \mathrm{CO}_{4}{ }^{2-}$, and $\mathrm{CO}_{4}{ }^{4-}$ (Figures 3, 6, and 9) have been determined as were structures

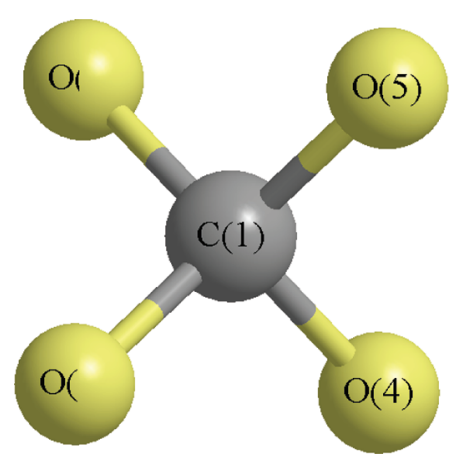

Figure 3. Tetrahedral $\mathrm{CO}_{4}{ }^{2-}$. All $\mathrm{C}-\mathrm{O}$ distances $=1.375 \AA$.

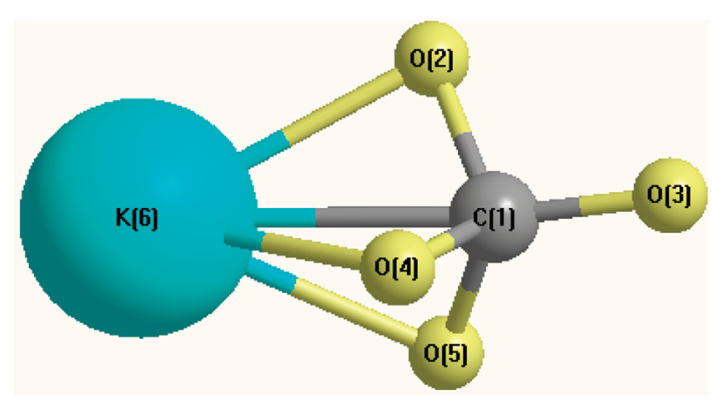

Figure 4. $\mathrm{KCO}_{4}^{-}$. Bond lengths: $\mathrm{K}-\mathrm{C} 1=2.630 \AA \AA \mathrm{K}-\mathrm{O} 2=\mathrm{K}-\mathrm{O} 4=$

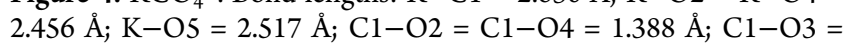
$1.330 \AA \AA \mathrm{A} ;-\mathrm{O} 5=1.393 \AA \AA$ A Bond angles: $\mathrm{K}-\mathrm{O} 4-\mathrm{C} 1=\mathrm{K}-\mathrm{O} 2-\mathrm{C} 1=$ $81.2^{\circ} ; \mathrm{K}-\mathrm{O} 5-\mathrm{C} 1=78.8^{\circ} ; \mathrm{O} 4-\mathrm{C} 1-\mathrm{O} 5=102.7^{\circ} ; \mathrm{O} 5-\mathrm{C} 1-\mathrm{O} 3=$ $118.7^{\circ} ; \mathrm{O} 4-\mathrm{C} 1-\mathrm{O} 2=114.9^{\circ} ; \mathrm{O} 3-\mathrm{C} 1-\mathrm{O} 2=109.0^{\circ} ; \mathrm{O} 4-\mathrm{C} 1-\mathrm{O} 5=$ $102.7^{\circ}$.

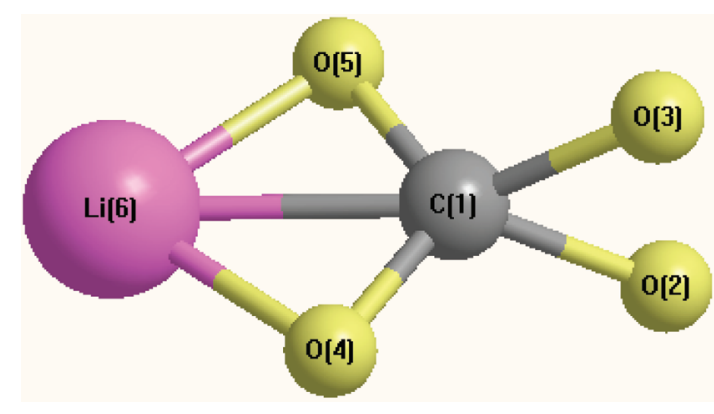

Figure 5. $\mathrm{LiCO}_{4}^{-}$. Bond lengths: $\mathrm{Li}-\mathrm{C} 1=2.028 \AA$; $\mathrm{Li}-\mathrm{O} 5=\mathrm{Li}-$ $\mathrm{O} 4=1.741 \AA$; $\mathrm{C} 1-\mathrm{O} 5=\mathrm{C} 1-\mathrm{O} 4=1.333 \AA$ 品 $1-\mathrm{O} 2=\mathrm{C} 1-\mathrm{O} 3=$ $1.450 \AA$ A. Bond angles: $\mathrm{O} 5-\mathrm{Li}-\mathrm{O} 4=81.1^{\circ} ; \mathrm{O} 5-\mathrm{C} 1-\mathrm{O} 4=116.1^{\circ}$; $\mathrm{O} 5-\mathrm{C} 1-\mathrm{O} 3=\mathrm{O} 2-\mathrm{C} 1-\mathrm{O} 4=116.8^{\circ} ; \mathrm{O} 3-\mathrm{C} 1-\mathrm{O} 2=63.1^{\circ}$.

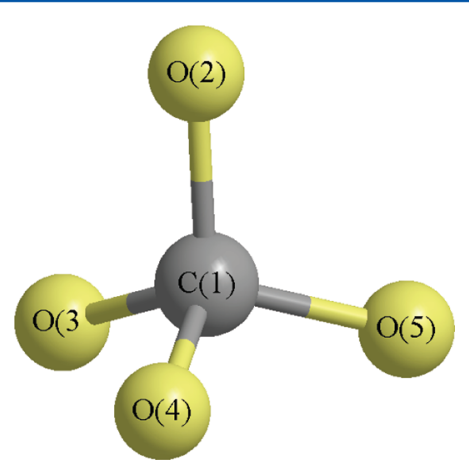

Figure 6. Tetrahedral $\mathrm{CO}_{4}{ }^{4-}$. All $\mathrm{C}-\mathrm{O}$ distances $=1.487 \AA$.

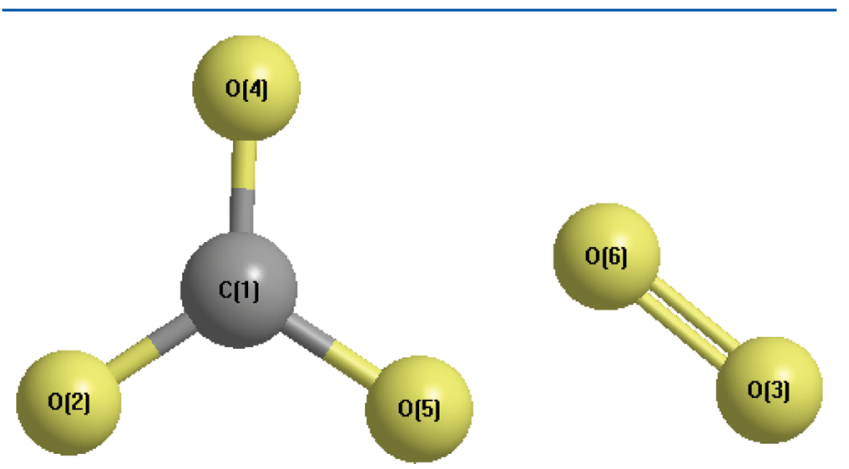

Figure 7. $\mathrm{CO}_{5}{ }^{2-}$. Carbonate-oxygen adduct. $\mathrm{C} 1-\mathrm{O} 2=1.277 \AA$ 的 $1-$

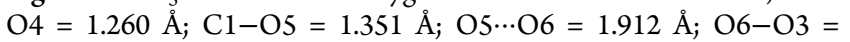
$1.315 \AA$. Both molecules lie in the same plane.

of an adduct of $\mathrm{CO}_{3}{ }^{2-}$ and $\mathrm{O}_{2}$ (Figure 7 ) and a dimer of $\mathrm{CO}_{3}{ }^{-}$ (Figure 8). The structures of $\mathrm{KCO}_{4}^{-}$and $\mathrm{LiCO}_{4}^{-}$are also shown (Figures 4 and 5) and will be discussed under the section Effect of $\mathrm{CO}_{2}$. A potential dimer of $\mathrm{CO}_{3}{ }^{2-}$ is theoretically unstable.

Cation Dependences. The monovalent cations, $\mathrm{K}^{+}, \mathrm{Li}^{+}$, and $\mathrm{Na}^{+}$form stable adducts with $\mathrm{CO}_{3}{ }^{2-}$ as expected (Figures 10-12). $\mathrm{K}^{+}$and $\mathrm{Na}^{+}$interact with two of the carbonate oxygens, whereas $\mathrm{Li}^{+}$interacts with a single oxygen (Figure 11). The highest occupied molecular orbitals (HOMOs) of these singly charged anions are shown in Figure 13.

The theoretical peroxide structures containing $\mathrm{K}^{+}, \mathrm{Li}^{+}$, or $\mathrm{Na}^{+}$are shown in Figures 14-21. There are triangular and linear forms for $\mathrm{KO}_{2}^{-}, \mathrm{LiO}_{2}^{-}$, and $\mathrm{NaO}_{2}^{-}$(Figures 14-19). There are also bent forms for $\mathrm{KO}_{2}^{-}$and $\mathrm{LiO}_{2}{ }^{-}$(Figures 20 and 21). Triangular and linear forms for $\mathrm{KO}_{2}$ and $\mathrm{LiO}_{2}$ are also possible (Figures 22-25). 

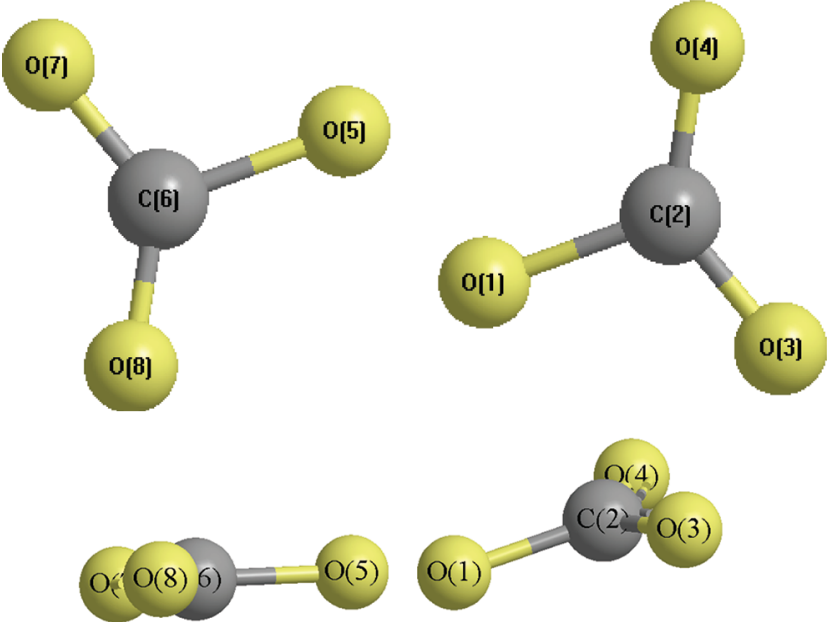

Figure 8. $\left(\mathrm{CO}_{3}^{-}\right)_{2}$. Bond lengths: $\mathrm{C} 6-\mathrm{O} 5=\mathrm{C} 2-\mathrm{O} 1=1.442 \AA$; 6 $\mathrm{O} 7=\mathrm{C} 2-\mathrm{O} 3=1.249 \AA ;$ C $6-\mathrm{O} 8=\mathrm{C} 2-\mathrm{O} 4=1.235 \AA ; 05 \cdots \mathrm{O} 1=$ $1.482 \AA$. This dimer is slightly off-planar as shown below.

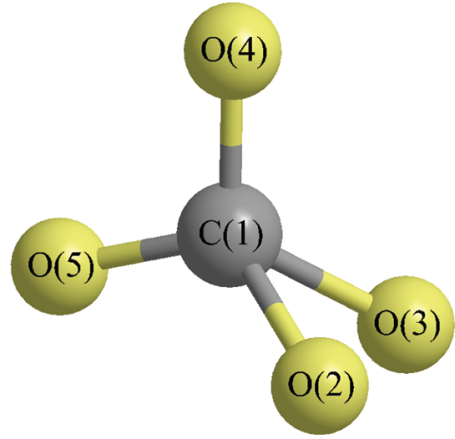

Figure 9. Distorted tetrahedral $\mathrm{CO}_{4}^{-}$. Bond lengths: $\mathrm{C} 1-\mathrm{O} 4=\mathrm{C} 1-$ $\mathrm{O} 5=1.299 \AA ; \mathrm{C} 1-\mathrm{O} 2=\mathrm{C} 1-\mathrm{O} 3=1.442 \AA$. Bond angles: $\mathrm{O} 2-\mathrm{C} 1-$ $\mathrm{O} 3=63.9^{\circ} ; \mathrm{O} 2-\mathrm{C} 1-\mathrm{O} 4=\mathrm{O} 2-\mathrm{C} 1-\mathrm{O} 5=\mathrm{O} 3-\mathrm{C} 1-\mathrm{O} 4=\mathrm{O} 3-\mathrm{C} 1-$ $\mathrm{O} 5=120.1^{\circ} ; \mathrm{O} 4-\mathrm{C} 1-\mathrm{O} 5=107.7^{\circ}$.
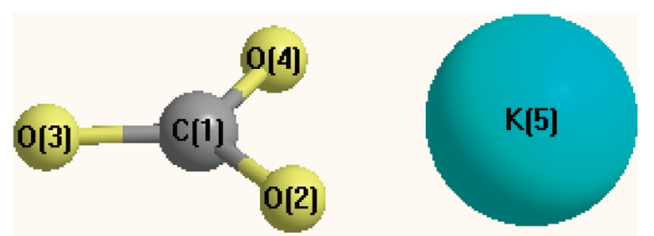

Figure 10. Planar $\mathrm{KCO}_{3}^{-}$. Bond lengths: $\mathrm{C} 1-\mathrm{O} 4=1.299 \AA \AA \mathrm{C} 1-$ $\mathrm{O} 2=\mathrm{C} 1-\mathrm{O} 3=1.300 \AA$ A. Other distances: $\mathrm{C} 1-\mathrm{K} 5=2.748 \AA \AA$; $\mathrm{K} 5-$ $\mathrm{O} 2=\mathrm{K} 5-\mathrm{O} 4=2.337 \AA$ A. Bond angles: $\mathrm{O} 4-\mathrm{C} 1-\mathrm{O} 3=\mathrm{O} 3-\mathrm{C} 1-\mathrm{O} 2=$ $121.9^{\circ} ; \mathrm{O} 4-\mathrm{C} 1-\mathrm{O} 2=116.3^{\circ}$.

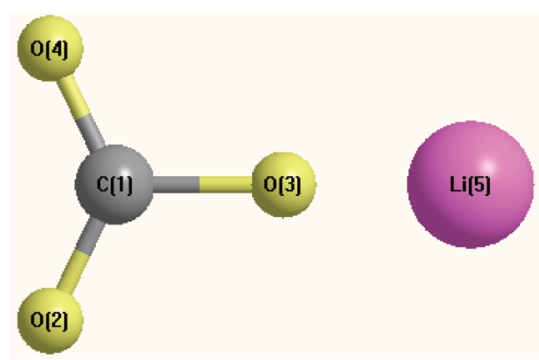

Figure 11. Planar $\mathrm{LiCO}_{3}^{-}$. Bond lengths: $\mathrm{C} 1-\mathrm{O} 4=\mathrm{C} 1-\mathrm{O} 2=1.252$ Aं; $\mathrm{C} 1-\mathrm{O} 3=1.403 \AA$. Other distances: $\mathrm{O} 3-\mathrm{Li} 5=1.561 \AA$. Bond angles: $\mathrm{O} 4-\mathrm{C} 1-\mathrm{O} 3=\mathrm{O} 3-\mathrm{C} 1-\mathrm{O} 2=115.5^{\circ} ; \mathrm{O} 4-\mathrm{C} 1-\mathrm{O} 2=129.1^{\circ}$.

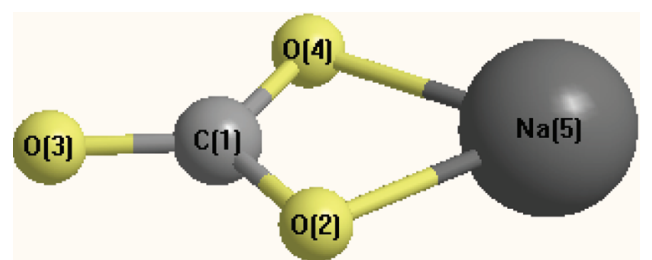

Figure 12. Planar $\mathrm{NaCO}_{3}^{-}$. Bond lengths: $\mathrm{C} 1-\mathrm{O} 4=\mathrm{C} 1-\mathrm{O} 2=$ $1.337 \AA$; $\mathrm{C} 1-\mathrm{O} 3=1.245 \AA$ 的 $1-\mathrm{Na} 5=2.447 \AA$. Other distances: $\mathrm{O} 4-\mathrm{Na} 5=\mathrm{O} 2-\mathrm{Na} 5=2.085 \AA$. Bond angles: $\mathrm{O} 4-\mathrm{C} 1-\mathrm{O} 3=\mathrm{O} 3-$ $\mathrm{C} 1-\mathrm{O} 2=121.6^{\circ} ; \mathrm{O} 4-\mathrm{C} 1-\mathrm{O} 2=116.8^{\circ}$.

(a)

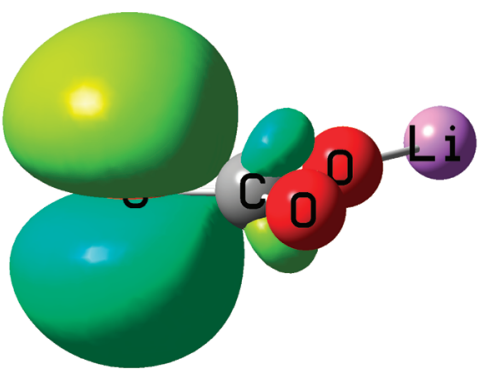

(b)

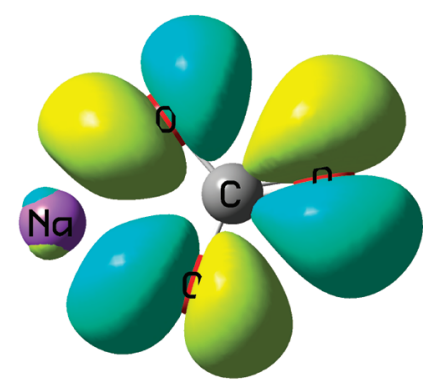

(c)

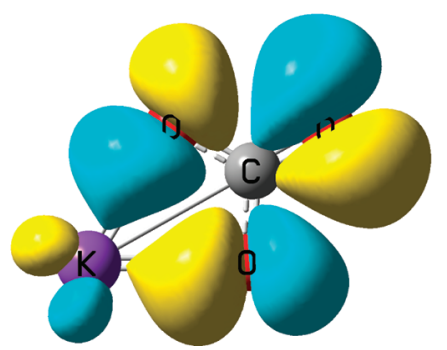

Figure 13. $\mathrm{B} 3 \mathrm{LYP} /(6-311+\mathrm{G}(2 \mathrm{~d}, \mathrm{p}))$ HOMOs of (a) $\mathrm{LiCO}_{3}^{-}$, (b) $\mathrm{NaCO}_{3}^{-}$, and (c) $\mathrm{KCO}_{3}^{-}$.

Absolute Energies. The B3LYP/ $(6-311+G(2 d, p))$ sum of electronic and thermal free energies (au's) of potential and known species at $923 \mathrm{~K}$ are shown in Table 2. There is no experimental evidence for certain of these species, despite their inclusion in Table 2. However, future investigations may change this conclusion.

Peroxide Structures. Of particular interest are the various structures of peroxide containing complex anions. The bent structure of $\mathrm{KO}_{2}{ }^{-}$is the lowest energy form by 5.8 and $10.5 \mathrm{kcal}$ in energy lower than the linear and triangular structures of $\mathrm{KO}_{2}{ }^{-}$at $923 \mathrm{~K}$ (Figures 21, 17, and 14). The triangular form of $\mathrm{LiO}_{2}{ }^{-}$is $45 \mathrm{kcal}$ lower in energy than the linear form of $\mathrm{LiO}_{2}{ }^{-}$ (Figures 15 and 18). The triangular and bent forms of $\mathrm{NaO}_{2}{ }^{-}$ 


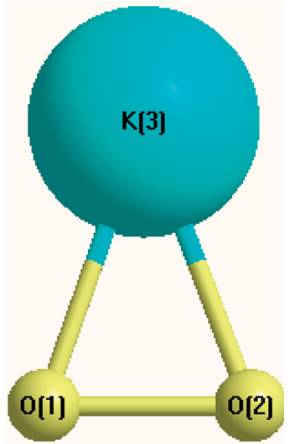

Figure 14. $\mathrm{B} 3 \mathrm{LYP} /(6-311+\mathrm{G}(2 \mathrm{~d}, \mathrm{p}))$ of $\mathrm{KO}_{2}^{-}$. Bond lengths: $\mathrm{K}-\mathrm{O} 1=$ $\mathrm{K}-\mathrm{O} 2=2.43 \AA ; \mathrm{O} 2-\mathrm{O} 1=1.38 \AA$. Bond angles: $\mathrm{K}-\mathrm{O} 2-\mathrm{O} 1=73.6^{\circ}$; $\mathrm{K}-\mathrm{O} 1-\mathrm{O} 2=73.3^{\circ} ; \mathrm{O} 2-\mathrm{K}-\mathrm{O} 1=39.1^{\circ}$.

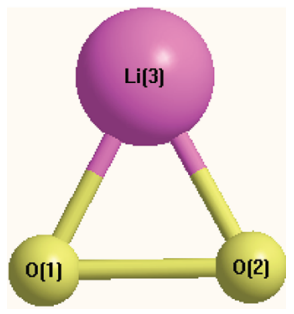

Figure 15. $\mathrm{B} 3 \mathrm{LYP} /(6-311+\mathrm{G}(2 \mathrm{~d}, \mathrm{p}))$ of $\mathrm{LiO}_{2}{ }^{-}$. Bond lengths: $\mathrm{Li}-$ $\mathrm{O} 1=\mathrm{Li}-\mathrm{O} 2=1.67 \AA$ 品 $2-\mathrm{O} 1=1.57 \AA$. Bond angles: $\mathrm{Li}-\mathrm{O} 2-\mathrm{O} 1=$ $\mathrm{Li}-\mathrm{O} 1-\mathrm{O} 2=61.8^{\circ} ; \mathrm{O} 2-\mathrm{Li}-\mathrm{O} 1=56.4^{\circ}$.

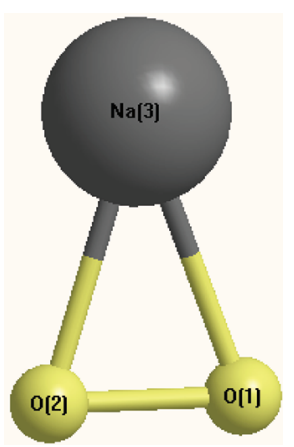

Figure 16. $\mathrm{B} 3 \mathrm{LYP} /(6-311+\mathrm{G}(2 \mathrm{~d}, \mathrm{p}))$ of $\mathrm{NaO}_{2}^{-}$. Bond lengths: $\mathrm{Na}-$ $\mathrm{O} 1=2.13 \AA ̊ \mathrm{Na}-\mathrm{O} 2=2.18 \AA ⿻ \mathrm{O} 2-\mathrm{O} 1=1.41 \AA$. . Bond angles: $\mathrm{Li}-$ $\mathrm{O} 2-\mathrm{O} 1=69.2^{\circ} ; \mathrm{Li}-\mathrm{O} 1-\mathrm{O} 2=72.7^{\circ} ; \mathrm{O} 2-\mathrm{Li}-\mathrm{O} 1=38.1^{\circ}$.

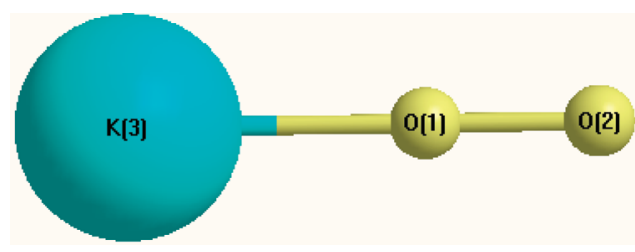

Figure 17. $\mathrm{B} 3 \mathrm{LYP} /(6-311+\mathrm{G}(2 \mathrm{~d}, \mathrm{p}))$ of linear $\mathrm{KO}_{2}^{-}$. Bond lengths: $\mathrm{K}-\mathrm{O} 1=2.29 \AA ; \mathrm{O} 1-\mathrm{O} 2=1.34 \AA$.

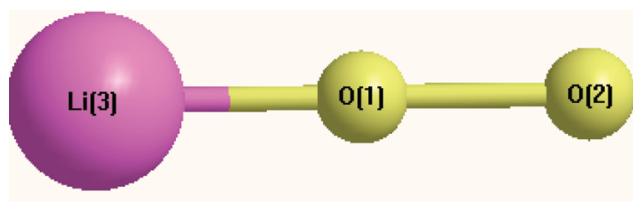

Figure 18. $\mathrm{B} 3 \mathrm{LYP} /(6-311+\mathrm{G}(2 \mathrm{~d}, \mathrm{p}))$ of linear $\mathrm{LiO}_{2}^{-}$. Bond lengths: $\mathrm{Li}-\mathrm{O} 1=1.59 \AA \AA \mathrm{O} 1-\mathrm{O} 2=1.36 \AA$.

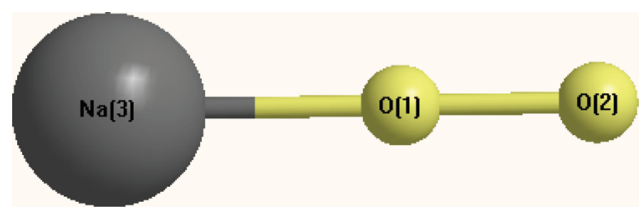

Figure 19. $\mathrm{B} 3 \mathrm{LYP} /(6-311+\mathrm{G}(2 \mathrm{~d}, \mathrm{p}))$ of linear $\mathrm{NaO}_{2}{ }^{-}$. Bond lengths: $\mathrm{Na}-\mathrm{O} 1=1.99 \AA ; \mathrm{O} 1-\mathrm{O} 2=1.34 \AA$.

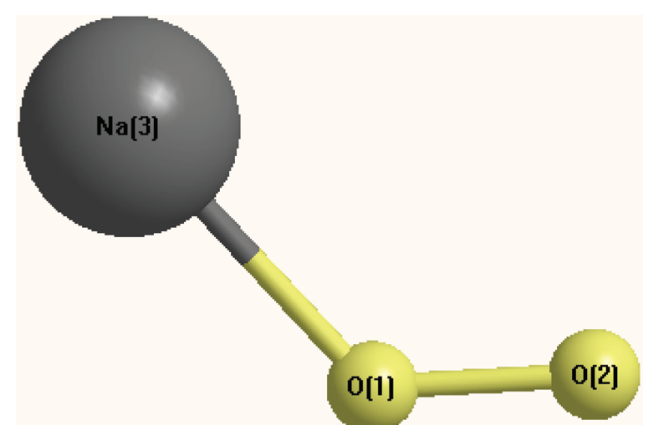

Figure 20. B3LYP $/(6-311+\mathrm{G}(2 \mathrm{~d}, \mathrm{p}))$ of bent $\mathrm{NaO}_{2}^{-}$. Bond lengths: $\mathrm{Na}-\mathrm{O} 1=2.16 \AA$; $\mathrm{O} 1-\mathrm{O} 2=1.34 \AA$. Bond angle: $\mathrm{Na}-\mathrm{O} 1-\mathrm{O} 2=$ $129.3^{\circ}$.

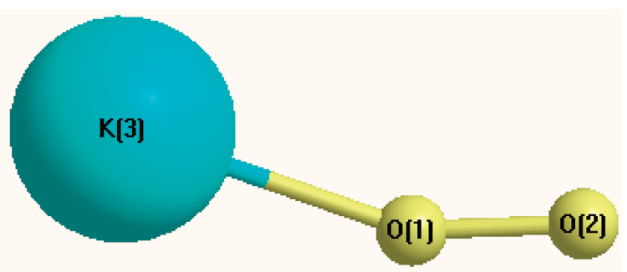

Figure 21. B3LYP/ $6-311+\mathrm{G}(2 \mathrm{~d}, \mathrm{p}))$ of bent $\mathrm{KO}_{2}{ }^{-}$. Bond lengths: $\mathrm{K}-$ $\mathrm{O} 1=2.37 \AA$ 品 $\mathrm{O} 1-\mathrm{O} 2=1.33 \AA$. Bond angle: $\mathrm{K}-\mathrm{O} 1-\mathrm{O} 2=158.5^{\circ}$.

are lower in energy than the linear form by approximately $25 \mathrm{kcal}$ at $923 \mathrm{~K}$ (Figures 16, 19, and 20).

There are single versions of $\mathrm{KCO}_{3}{ }^{-}, \mathrm{LiCO}_{3}{ }^{-}$, and $\mathrm{NaCO}_{3}{ }^{-}$at $923 \mathrm{~K}$ (Figures 10-12). Potentially stable forms of $\mathrm{CO}_{4}{ }^{2-}$, $\mathrm{CO}_{4}{ }^{4-}$, and $\mathrm{CO}_{4}{ }^{-}$are shown in Figures 3, 6, and 9. A dimer of $\mathrm{CO}_{3}^{-}\left(\mathrm{C}_{2} \mathrm{O}_{6}{ }^{2-}\right)$ may exist (Figure 8) as does an adduct of $\mathrm{CO}_{3}{ }^{2-}$ and $\mathrm{O}_{2}\left(\mathrm{CO}_{5}{ }^{2-}\right)$ (Figure 7 ).

Assignment of Raman Bands. The assignments in this section are made on the same basis as those reported in previous Raman analysis of ionic liquids. ${ }^{14-16}$ The calculated Raman frequencies were seen to match the experimental results to within 6\%, as shown in Figure 26. ${ }^{14-16}$ This approach rules out several anions (including free carbonate) that are not coordinated to an individual monovalent cation in the high temperature carbonate melts. Species other than carbonate are also seen to require coordination by a monovalent cation.

Boyd and co-workers ${ }^{1}$ reported the Raman spectrum of pure molten $\mathrm{Li}_{2} \mathrm{CO}_{3}$ at $492 \mathrm{~K}$ and reported a strong polarized band at $1072 \mathrm{~cm}^{-1}$. This is assigned to the $\mathrm{C}-\mathrm{O}-\mathrm{Li}$ symmetric stretch vibration of $\mathrm{LiCO}_{3}^{-}$at $1124 \mathrm{~cm}^{-1}$ (Table 1). A weaker band observed at $1752 \mathrm{~cm}^{-1}$ is assigned to the $\mathrm{O}-\mathrm{C}-\mathrm{O}$ inplane asymmetric stretch at $1806 \mathrm{~cm}^{-1}$ in Table 1 . In a separate article, Itoh and co-workers ${ }^{3}$ reported the spectrum of $\mathrm{Li}_{2} \mathrm{CO}_{3}$ at $1123 \mathrm{~K}$ in an $\mathrm{O}_{2} / \mathrm{CO}_{2}(90 / 10)$ atmosphere. They observed Raman bands at 613,805 , and $1065 \mathrm{~cm}^{-1}$. The Raman band at $1065 \mathrm{~cm}^{-1}$ (not observed by Boyd et al. ${ }^{1}$ ) is tentatively assigned to the $\mathrm{C}-\mathrm{O}-\mathrm{Li}$ symmetric stretch band at $1124 \mathrm{~cm}^{-1}$ with a possible contribution at $1081 \mathrm{~cm}^{-1}$ from the higher energy 


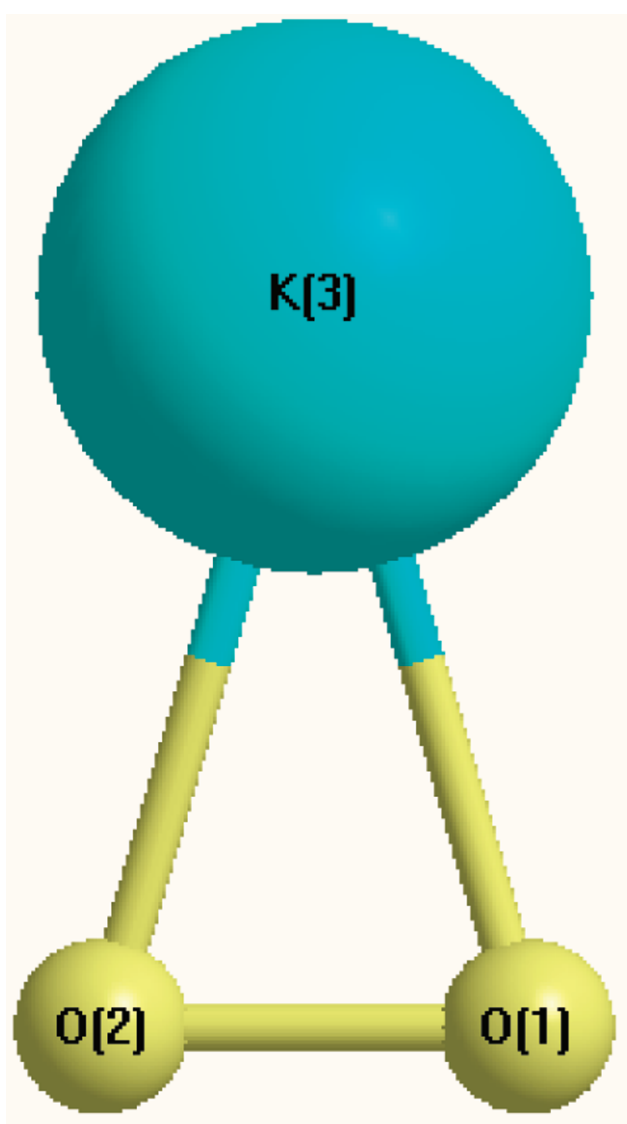

Figure 22. $\mathrm{B} 3 \mathrm{LYP} /(6-311+\mathrm{G}(2 \mathrm{~d}, \mathrm{p}))$ of triangular $\mathrm{KO}_{2}$. Bond lengths: $\mathrm{K}-\mathrm{O} 1=\mathrm{K}-\mathrm{O} 2=2.39 \AA$; $\mathrm{O} 1-\mathrm{O} 2=1.34 \AA$. Bond angles: $\mathrm{K}-\mathrm{O} 1-\mathrm{O} 2=$ $\mathrm{K}-\mathrm{O} 2-\mathrm{O} 1=73.7^{\circ} ; \mathrm{O} 2-\mathrm{K}-\mathrm{O} 1=32.6^{\circ}$.

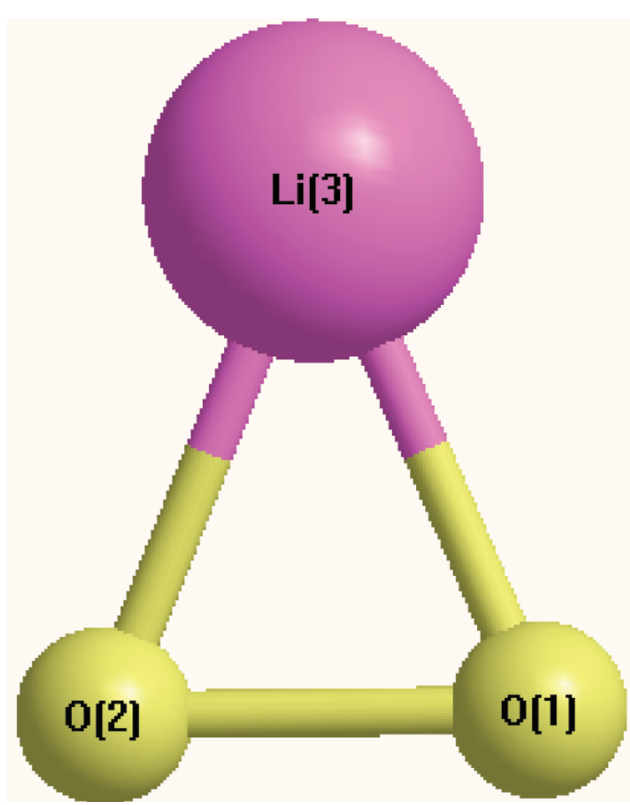

Figure 23. $\mathrm{B} 3 \mathrm{LYP} /(6-311+\mathrm{G}(2 \mathrm{~d}, \mathrm{p}))$ of triangular $\mathrm{LiO}_{2}$. Bond lengths: $\mathrm{Li}-\mathrm{O} 1=\mathrm{Li}-\mathrm{O} 2=1.75 \AA ̊ 010$ $\mathrm{O} 2=\mathrm{Li}-\mathrm{O} 2-\mathrm{O} 1=67.3^{\circ} ; \mathrm{O} 2-\mathrm{Li}-\mathrm{O} 1=45.3^{\circ}$.

linear forms of $\mathrm{LiO}_{2}^{-}$(Table 1). The Raman frequency at $805 \mathrm{~cm}^{-1}$ is assigned to the triangular $\mathrm{KO}_{2}^{-} \mathrm{O}-\mathrm{K}-\mathrm{O}$ symmetric bend frequency at $804 \mathrm{~cm}^{-1}$ as discussed under

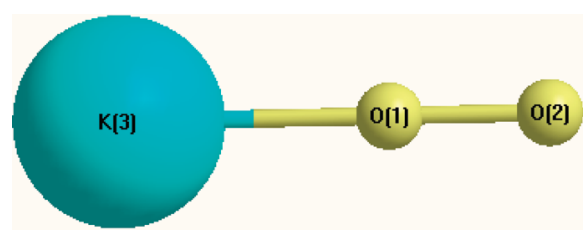

Figure 24. $\mathrm{B} 3 \mathrm{LYP} /(6-311+\mathrm{G}(2 \mathrm{~d}, \mathrm{p}))$ of linear $\mathrm{KO}_{2}$. Bond lengths: $\mathrm{K}-\mathrm{O} 1=2.23 \AA ; \mathrm{O} 1-\mathrm{O} 2=1.32 \AA$.

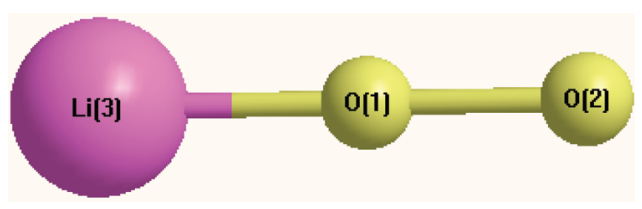

Figure 25. $\mathrm{B} 3 \mathrm{LYP} /(6-311+\mathrm{G}(2 \mathrm{~d}, \mathrm{p}))$ of linear $\mathrm{LiO}_{2}$. Bond lengths: $\mathrm{Li}-\mathrm{O} 1=1.61 \AA ; \mathrm{O} 1-\mathrm{O} 2=1.31 \AA$.

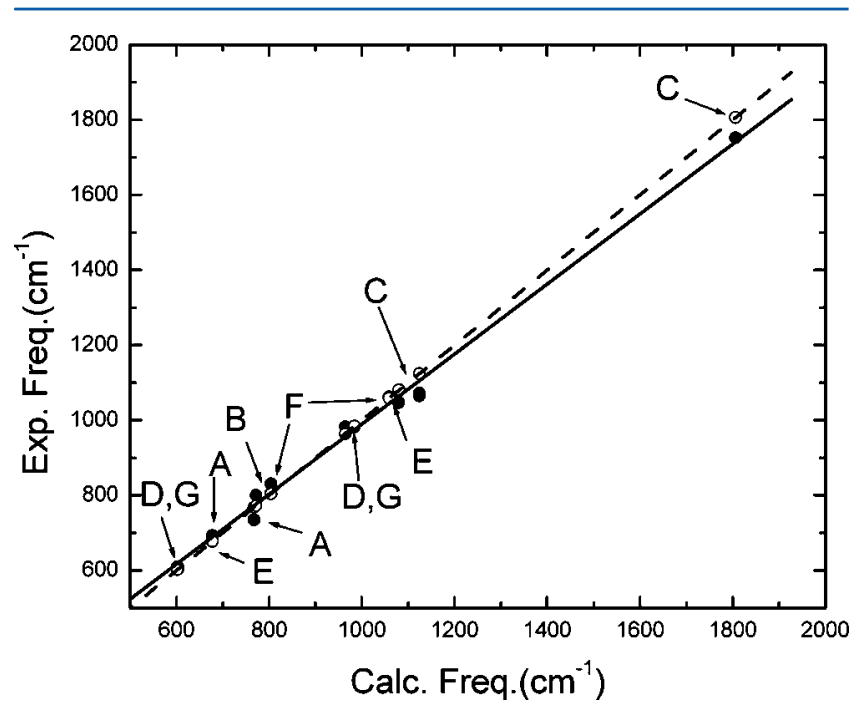

Figure 26. Experimental frequency $\left(\mathrm{cm}^{-1}\right)$ vs calculated frequency $\left(\mathrm{cm}^{-1}\right)$ for molten carbonate species: $\mathrm{A}, \mathrm{LiO}_{2} ; \mathrm{B}, \mathrm{KO}_{2}^{-} ; \mathrm{C}, \mathrm{LiCO}_{3}{ }^{-} ; \mathrm{D}$, $\mathrm{CO}_{4}{ }^{2-} ; \mathrm{E}, \mathrm{KCO}_{3}^{-} ; \mathrm{F}, \mathrm{LiO}_{2}^{-} ; \mathrm{G}, \mathrm{CO}_{4}{ }^{2-}, \mathrm{KCO}_{4}^{-}$. Dashed line $($ theoretical correlation $)=$ slope $=1.00$; solid line slope $=0.94(R=$ 0.997).

the section Peroxides. The assignment of the Raman band at $613 \mathrm{~cm}^{-1}$ is assigned in the peroxide section that follows.

Peroxides. Lin and co-workers ${ }^{2}$ report a series of experiments in which molten $\mathrm{Li} / \mathrm{K}$ eutectic carbonate solutions in the presence of either $\mathrm{O}_{2}$ or Ar were subjected to a wide range of temperatures. When $\mathrm{O}_{2}$ is present, one observes a sharp peak at $1062 \mathrm{~cm}^{-1}$ and a weaker broad peak at $830 \mathrm{~cm}^{-1}$. The Raman peak at $830 \mathrm{~cm}^{-1}$ is absent in the presence of $\mathrm{Ar}$, and the peak at $1062 \mathrm{~cm}^{-1}$ shifts to $1058 \mathrm{~cm}^{-1}$. The authors assign the $830 \mathrm{~cm}^{-1}$ band to peroxide produced via eq 1 :

$$
\mathrm{O}_{2}+2 \mathrm{CO}_{3}^{2-} \leftrightarrows 2 \mathrm{O}_{2}^{2-}+2 \mathrm{CO}_{2}
$$

The above gas phase reaction, eq 1 , is quite endothermic $\left(\Delta G^{\circ}=+218 \mathrm{kcal} / \mathrm{mol}\right)$; however, when $\mathrm{K}^{+}$ions are added to form $\mathrm{KO}_{2}^{-}$, the overall process becomes exothermic $\left(\Delta G^{\circ} \approx\right.$ -202 to $-207 \mathrm{kcal} / \mathrm{mol}$; see Table 2). The addition of $\mathrm{Li}^{+}$ ions to form $\mathrm{LiO}_{2}{ }^{-}$is even more exothermic $\left(\Delta G^{\circ}=-261\right.$ to $-284 \mathrm{kcal} / \mathrm{mol}$; see Table 2$)$. In view of the above, the broad band at $830 \mathrm{~cm}^{-1}$ is assigned to the strong theoretical band at $804 \mathrm{~cm}^{-1}$ for the triangular version of $\mathrm{KO}_{2}^{-}$in Table 1. This assignment is made even though the triangular form of $\mathrm{KO}_{2}{ }^{-}$is 
Table 1. B3LYP/(6-311+G(d,p)) Vibrational Assignments $\left(\mathrm{cm}^{-1}\right)$

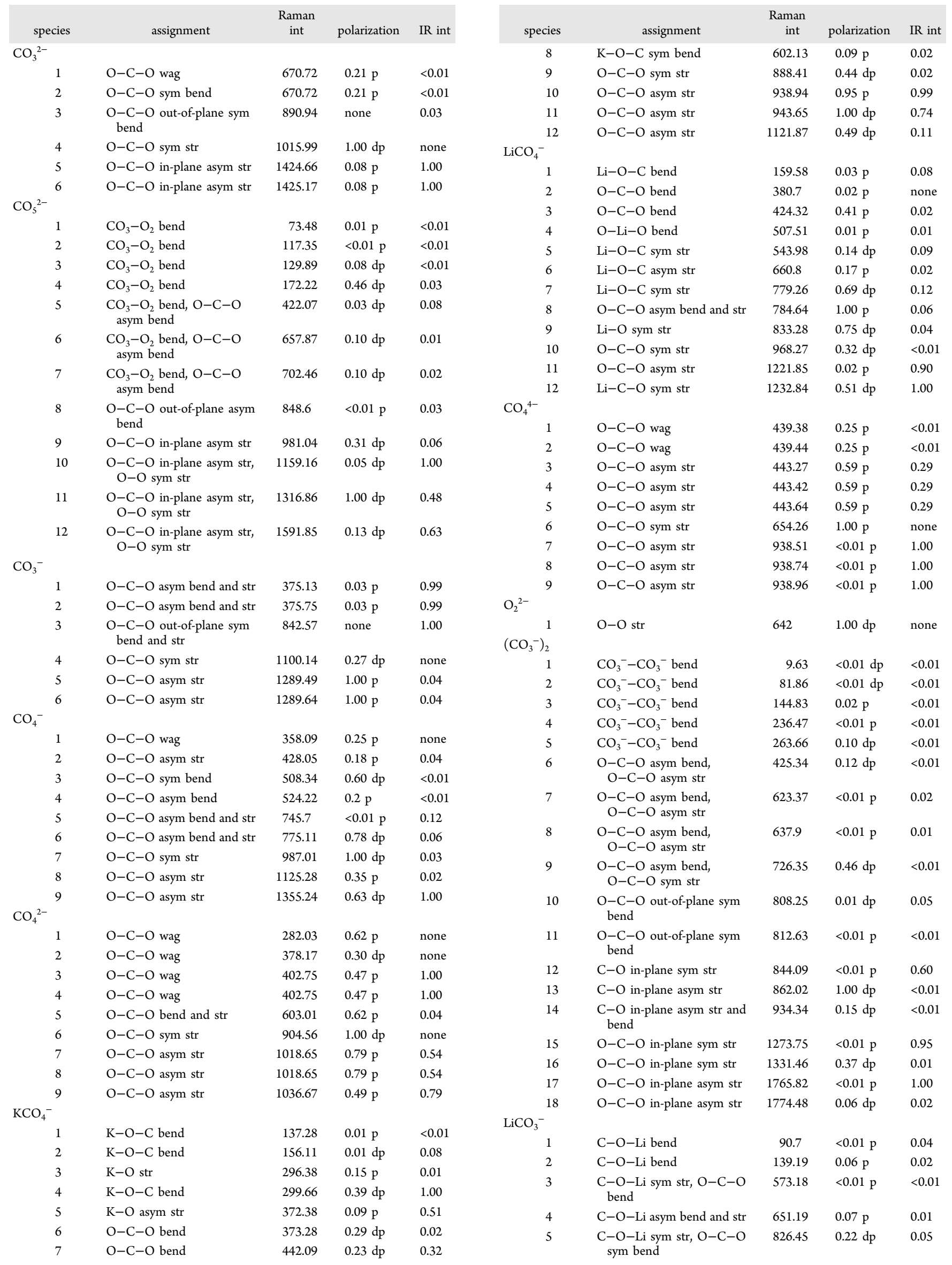


Table 1. continued

\begin{tabular}{|c|c|c|c|c|}
\hline species & assignment & $\begin{array}{c}\text { Raman } \\
\text { int }\end{array}$ & polarization & IR int \\
\hline 6 & $\begin{array}{l}\mathrm{C}-\mathrm{O}-\mathrm{Li} \text { asym str, } \mathrm{O}-\mathrm{C}-\mathrm{O} \\
\text { asym bend }\end{array}$ & 845.11 & $<0.01 \mathrm{p}$ & 0.01 \\
\hline 7 & $\mathrm{C}-\mathrm{O}-\mathrm{Li}$ sym str & 1124.14 & $0.45 \mathrm{dp}$ & 0.13 \\
\hline 8 & $\mathrm{O}-\mathrm{C}-\mathrm{O}$ in-plane sym str & 1276.24 & $0.28 \mathrm{dp}$ & 0.24 \\
\hline 9 & $\mathrm{O}-\mathrm{C}-\mathrm{O}$ in-plane asym str & 1805.63 & $1.00 \mathrm{p}$ & 1.00 \\
\hline \multicolumn{5}{|l|}{$\mathrm{KCO}_{3}^{-}$} \\
\hline 1 & $\begin{array}{l}\mathrm{K} \text {-carbonate out-of-plane } \\
\text { bend }\end{array}$ & 67.54 & $<0.01 \mathrm{p}$ & 0.01 \\
\hline 2 & $\mathrm{~K}$-carbonate in-plane bend & 266.73 & $<0.01 \mathrm{p}$ & 0.02 \\
\hline 3 & $\mathrm{~K}$-carbonate in-plane str & 324.83 & $0.22 \mathrm{dp}$ & 0.09 \\
\hline 4 & $\begin{array}{l}\mathrm{O}-\mathrm{C}-\mathrm{O} \text { in-plane asym } \\
\text { bend }\end{array}$ & 615.01 & $0.15 \mathrm{p}$ & 0.01 \\
\hline 5 & $\mathrm{O}-\mathrm{C}-\mathrm{O}$ in-plane sym bend & 678.07 & $0.37 \mathrm{dp}$ & 0.01 \\
\hline 6 & C out-of-plane inversion & 852.08 & $0.01 \mathrm{p}$ & 0.01 \\
\hline 7 & $\mathrm{O}-\mathrm{C}-\mathrm{O}$ in-plane sym str & 994.25 & $0.26 \mathrm{dp}$ & 0.05 \\
\hline 8 & $\mathrm{O}-\mathrm{C}-\mathrm{O}$ in-plane asym str & 1058.77 & $1.00 \mathrm{p}$ & 0.14 \\
\hline 9 & $\mathrm{O}-\mathrm{C}-\mathrm{O}$ in-plane sym str & 1577.42 & $0.98 \mathrm{dp}$ & 1.00 \\
\hline \multicolumn{5}{|l|}{$\mathrm{NaCO}_{3}^{-}$} \\
\hline 1 & $\begin{array}{l}\mathrm{Na}-\text { carbonate out-of-plane } \\
\text { bend }\end{array}$ & 104.42 & $<0.01 \mathrm{p}$ & 0.03 \\
\hline 2 & $\mathrm{Na}-\mathrm{O}$ asym str & 358.4 & $0.03 \mathrm{p}$ & $<0.01$ \\
\hline 3 & $\mathrm{Na}-\mathrm{O}$ sym str & 399.51 & $0.03 \mathrm{dp}$ & 0.06 \\
\hline 4 & $\begin{array}{l}\mathrm{O}-\mathrm{C}-\mathrm{O} \text { wag, } \mathrm{Na}-\mathrm{O} \text { asym } \\
\text { str }\end{array}$ & 639.26 & $0.35 \mathrm{p}$ & 0.03 \\
\hline 5 & $\begin{array}{l}\mathrm{O}-\mathrm{C}-\mathrm{O} \text { wag, } \mathrm{Na}-\mathrm{O} \text { sym } \\
\text { str }\end{array}$ & 709.21 & $0.05 \mathrm{dp}$ & $<0.01$ \\
\hline 6 & $\mathrm{O}-\mathrm{C}-\mathrm{O}$ out-of-plane bend & 846.77 & $0.02 \mathrm{p}$ & 0.01 \\
\hline 7 & $\mathrm{O}-\mathrm{C}-\mathrm{O}$ sym str & 977.93 & $0.02 \mathrm{dp}$ & 0.04 \\
\hline 8 & $\mathrm{O}-\mathrm{C}-\mathrm{O}$ asym str & 1086.42 & $1.00 \mathrm{p}$ & 1.00 \\
\hline 9 & $\mathrm{O}-\mathrm{C}-\mathrm{O}$ sym str & 1584.13 & $0.18 \mathrm{dp}$ & 0.57 \\
\hline \multicolumn{5}{|l|}{$\begin{array}{l}\mathrm{NaO}_{2}^{-} \text {, trian- } \\
\text { gular form }\end{array}$} \\
\hline 1 & $\mathrm{Na}-\mathrm{O}-\mathrm{O}$ bend & 267.59 & $0.02 \mathrm{dp}$ & 0.01 \\
\hline 2 & $\mathrm{Na}-\mathrm{O}$ str & 371.47 & $0.09 \mathrm{dp}$ & 0.05 \\
\hline 3 & $\mathrm{O}-\mathrm{O}$ str & 918.84 & $1.00 \mathrm{dp}$ & 1.00 \\
\hline \multicolumn{5}{|l|}{$\begin{array}{l}\mathrm{NaO}_{2}^{-} \text {, linear } \\
\text { form }\end{array}$} \\
\hline 1 & $\mathrm{Na}-\mathrm{O}-\mathrm{O}$ bend & 151.57 & $<0.01 \mathrm{p}$ & 0.13 \\
\hline 2 & $\mathrm{Na}-\mathrm{O}-\mathrm{O}$ bend & 151.57 & $<0.01 \mathrm{p}$ & 0.13 \\
\hline 3 & $\mathrm{Na}-\mathrm{O}$ str & 414.75 & $<0.01 \mathrm{dp}$ & 0.01 \\
\hline 4 & $\mathrm{Na}-\mathrm{O}-\mathrm{O}$ asym str & 1083.74 & $1.00 \mathrm{dp}$ & 1.00 \\
\hline \multicolumn{5}{|l|}{$\begin{array}{l}\mathrm{NaO}_{2}^{-} \text {, angu- } \\
\text { lar form }\end{array}$} \\
\hline 1 & $\mathrm{Na}-\mathrm{O}-\mathrm{O}$ bend & 101.93 & $0.02 \mathrm{dp}$ & 0.01 \\
\hline 2 & $\mathrm{Na}-\mathrm{O}$ str & 314.62 & $0.01 \mathrm{dp}$ & $<0.01$ \\
\hline 3 & $\mathrm{O}-\mathrm{O}$ str & 1096.28 & $1.00 \mathrm{dp}$ & 1.00 \\
\hline \multicolumn{5}{|l|}{$\begin{array}{l}\mathrm{LiO}_{2}^{-}, \text {trian- } \\
\text { gular form }\end{array}$} \\
\hline 1 & $\mathrm{O}-\mathrm{Li}-\mathrm{O}$ asym str & 577.56 & $0.56 \mathrm{dp}$ & 1.00 \\
\hline 2 & $\mathrm{O}-\mathrm{Li}-\mathrm{O}$ sym bend & 645.48 & $1.00 \mathrm{dp}$ & $<0.01$ \\
\hline 3 & $\mathrm{O}-\mathrm{Li}-\mathrm{O}$ sym str & 949.3 & $0.06 \mathrm{dp}$ & 0.03 \\
\hline
\end{tabular}

theoretically the highest energy form as indicated in Table 2. There are no strong theoretical $\mathrm{LiO}_{2}{ }^{-}$bands that match the observed $830 \mathrm{~cm}^{-1}$ band. The strong band at $1062 \mathrm{~cm}^{-1}$ is likely due to the strong $\mathrm{KCO}_{3}^{-}$band at $1059 \mathrm{~cm}^{-1}$ in Table 1 . One cannot completely rule out the possibility that the $\mathrm{LiCO}_{3}{ }^{-}$ band at $1124 \mathrm{~cm}^{-1}$ also contributes. The weak band at $692 \mathrm{~cm}^{-1}$ is present in an atmosphere of either $\mathrm{O}_{2}$ or $\mathrm{Ar}$ and is assigned to the Raman vibration of $\mathrm{KCO}_{3}{ }^{-}$at $678 \mathrm{~cm}^{-1}$.

Effect of $\mathrm{CO}_{2}$. Chen and co-workers ${ }^{4}$ reported the effect of $\mathrm{CO}_{2}$ on molten $\mathrm{Li} / \mathrm{K}$ carbonate at $923 \mathrm{~K}$. The $\mathrm{Li} / \mathrm{K}$ carbonate melt under $1 \mathrm{~atm}$ of $\mathrm{O}_{2}$ produces initial Raman peaks at 620, 832, and $1062 \mathrm{~cm}^{-1}$. As time progresses, a weak band at

\begin{tabular}{|c|c|c|c|c|}
\hline species & assignment & $\begin{array}{c}\text { Raman } \\
\text { int }\end{array}$ & polarization & IR int \\
\hline \multicolumn{5}{|c|}{$\begin{array}{l}\mathrm{LiO}_{2}^{-} \text {, linear } \\
\text { form }\end{array}$} \\
\hline 1 & $\mathrm{Li}-\mathrm{O}-\mathrm{O}$ bend & 293.93 & $<0.01 \mathrm{p}$ & 0.01 \\
\hline 2 & $\mathrm{Li}-\mathrm{O}-\mathrm{O}$ bend & 293.93 & $<0.01 \mathrm{p}$ & 0.01 \\
\hline 3 & $\mathrm{Li}-\mathrm{O}-\mathrm{O}$ sym str & 771.85 & $0.03 \mathrm{dp}$ & 0.13 \\
\hline 4 & $\mathrm{Li}-\mathrm{O}-\mathrm{O}$ asym str & 1081.12 & $1.00 \mathrm{dp}$ & 1.00 \\
\hline \multicolumn{5}{|c|}{$\begin{array}{l}\mathrm{KO}_{2}^{-} \text {, triangu- } \\
\text { lar form }\end{array}$} \\
\hline 1 & $\mathrm{O}-\mathrm{K}-\mathrm{O}$ asym str & 328.85 & $0.02 \mathrm{p}$ & 0.01 \\
\hline 2 & $\mathrm{O}-\mathrm{K}-\mathrm{O}$ sym str & 381.03 & $0.20 \mathrm{dp}$ & $<0.01$ \\
\hline 3 & $\mathrm{O}-\mathrm{K}-\mathrm{O}$ sym bend & 803.98 & $1.00 \mathrm{dp}$ & 1.00 \\
\hline \multicolumn{5}{|l|}{$\begin{array}{l}\mathrm{KO}_{2}^{-} \text {, bent } \\
\text { form }\end{array}$} \\
\hline 1 & $\mathrm{O}-\mathrm{K}-\mathrm{O}$ asym str & 95.25 & $0.03 \mathrm{dp}$ & $<0.01$ \\
\hline 2 & $\mathrm{O}-\mathrm{K}-\mathrm{O}$ sym str & 252.47 & $0.01 \mathrm{p}$ & $<0.01$ \\
\hline 3 & $\mathrm{O}-\mathrm{K}-\mathrm{O}$ sym bend & 1126.03 & $1.00 \mathrm{dp}$ & 1.00 \\
\hline \multicolumn{5}{|c|}{$\begin{array}{l}\mathrm{KO}_{2}^{-} \text {, linear } \\
\text { form }\end{array}$} \\
\hline 1 & $\mathrm{~K}-\mathrm{O}-\mathrm{O}$ bend & 142.72 & $0.04 \mathrm{p}$ & 0.06 \\
\hline 2 & $\mathrm{~K}-\mathrm{O}-\mathrm{O}$ bend & 142.72 & $0.04 \mathrm{p}$ & 0.06 \\
\hline 3 & $\mathrm{~K}-\mathrm{O}$ str & 306.64 & $0.04 \mathrm{dp}$ & $<0.01$ \\
\hline 4 & $\mathrm{~K}-\mathrm{O}-\mathrm{O}$ asym str & 1080.29 & $1.00 \mathrm{dp}$ & 1.00 \\
\hline \multicolumn{5}{|c|}{$\begin{array}{l}\mathrm{LiO}_{2} \text {, triangu- } \\
\text { lar form }\end{array}$} \\
\hline 1 & $\mathrm{O}-\mathrm{Li}-\mathrm{O}$ asym str & 539.17 & $0.09 \mathrm{p}$ & 0.25 \\
\hline 2 & $\mathrm{O}-\mathrm{Li}-\mathrm{O}$ sym str & 768.32 & $1.00 \mathrm{dp}$ & 1.00 \\
\hline 3 & $\mathrm{O}-\mathrm{O}$ sym str & 1174.79 & $0.67 \mathrm{dp}$ & 0.08 \\
\hline \multicolumn{5}{|l|}{$\begin{array}{l}\mathrm{LiO}_{2} \text {, linear } \\
\text { form }\end{array}$} \\
\hline 1 & $\mathrm{Li}-\mathrm{O}-\mathrm{O}$ bend & 125.92 & $<0.01 \mathrm{p}$ & 0.34 \\
\hline 2 & $\mathrm{Li}-\mathrm{O}-\mathrm{O}$ asym str & 177.28 & $0.03 \mathrm{p}$ & 0.36 \\
\hline 3 & $\mathrm{Li}-\mathrm{O}-\mathrm{O}$ sym str & 775.35 & $0.08 \mathrm{dp}$ & 1.00 \\
\hline 4 & $\mathrm{Li}-\mathrm{O}-\mathrm{O}$ asym str & 1323.94 & $1.00 \mathrm{dp}$ & 0.17 \\
\hline \multicolumn{5}{|c|}{$\begin{array}{l}\mathrm{NaO}_{2} \text {, triangu- } \\
\text { lar form }\end{array}$} \\
\hline 1 & $\mathrm{Na}-\mathrm{O}-\mathrm{O}$ asym str & 368.49 & $1.00 \mathrm{p}$ & 0.04 \\
\hline 2 & $\mathrm{Na}-\mathrm{O}-\mathrm{O}$ sym str & 436.32 & $0.92 \mathrm{dp}$ & 1.00 \\
\hline 3 & $\mathrm{O}-\mathrm{O}$ sym str & 1167.71 & $0.60 \mathrm{dp}$ & 0.02 \\
\hline \multicolumn{5}{|c|}{$\begin{array}{l}\mathrm{KO}_{2} \text {, triangu- } \\
\text { lar form }\end{array}$} \\
\hline 1 & $\mathrm{O}-\mathrm{K}-\mathrm{O}$ asym str & 332.39 & $0.03 \mathrm{p}$ & 0.08 \\
\hline 2 & $\mathrm{O}-\mathrm{K}-\mathrm{O}$ sym str & 353.69 & $0.16 \mathrm{dp}$ & 1.00 \\
\hline 3 & $\mathrm{O}-\mathrm{K}-\mathrm{O}$ sym bend & 1193.24 & $1.00 \mathrm{dp}$ & 0.05 \\
\hline \multicolumn{5}{|l|}{$\begin{array}{l}\mathrm{KO}_{2} \text {, linear } \\
\text { form }\end{array}$} \\
\hline 1 & $\mathrm{~K}-\mathrm{O}-\mathrm{O}$ bend & 90.54 & $<0.01 \mathrm{p}$ & 0.06 \\
\hline 2 & $\mathrm{~K}-\mathrm{O}-\mathrm{O}$ bend & 121.68 & $0.09 \mathrm{p}$ & 0.06 \\
\hline 3 & $\mathrm{~K}-\mathrm{O}$ str & 347.96 & $0.09 \mathrm{dp}$ & $<0.01$ \\
\hline 4 & $\mathrm{~K}-\mathrm{O}-\mathrm{O}$ asym str & 1252.9 & $1.00 \mathrm{dp}$ & 1.00 \\
\hline $\mathrm{O}_{2}$ & $\mathrm{O}-\mathrm{O}$ str & 1194.17 & $1.00 \mathrm{dp}$ & none \\
\hline
\end{tabular}

$982 \mathrm{~cm}^{-1}$ is observed. Upon addition of $\mathrm{CO}_{2}$ to the $\mathrm{O}_{2}$ atmosphere in the $\mathrm{Li} / \mathrm{K}$ carbonate melt, the $832 \mathrm{~cm}^{-1}$ peak decreases while the peaks at 620 and $982 \mathrm{~cm}^{-1}$ grow. The peak at $1062 \mathrm{~cm}^{-1}$ remains essentially constant. The authors ${ }^{4}$ suggest that the addition of $\mathrm{CO}_{2}$ leads to the formation of $\mathrm{CO}_{4}{ }^{2-}$ via eq 2 :

$$
\mathrm{CO}_{2}+\mathrm{O}_{2}^{2-} \leftrightarrows \mathrm{CO}_{4}^{2-} \quad(\Delta G=-48.9 \mathrm{kcal})
$$

This prediction is supported by several Raman bands. The broad peak at $982 \mathrm{~cm}^{-1}$ is well represented by the strong Raman vibrations 6-9 (905-1037 $\mathrm{cm}^{-1}$ ) for $\mathrm{CO}_{4}{ }^{2-}$ as given in Table 1 . The broad Raman peak at approximately $610 \mathrm{~cm}^{-1}$ is 
Table 2. B3LYP/(6-311+G(d,p)) Sum of Electronic and Thermal Free Energies at $923 \mathrm{~K}$ and 1 atm

\begin{tabular}{ll}
\multicolumn{1}{c}{ species } & $G^{\circ a}(\mathrm{au})^{b}$ \\
$\mathrm{O}_{2}{ }^{-}$ & -150.452141 \\
$\mathrm{C}_{2} \mathrm{O}_{6}{ }^{2-}$ & -527.812642 \\
$\mathrm{CO}_{4}{ }^{2-}$ & -338.916608 \\
$\mathrm{CO}_{4}{ }^{--}$ & -337.874808 \\
$\mathrm{KCO}_{4}{ }^{-}$ & -939.105058 \\
$\mathrm{LiCO}_{4}{ }^{-}$ & -346.762904 \\
$\mathrm{O}^{2-}$ & -75.052277 \\
triangular $\mathrm{KO}_{2}{ }^{-}$ & -750.430000 \\
linear $\mathrm{KO}_{2}{ }^{-}$ & -750.437512 \\
bent $\mathrm{KO}_{2}{ }^{-}$ & -750.446818 \\
triangular $\mathrm{LiO}_{2}{ }^{-}$ & -158.074821 \\
linear $\mathrm{LiO}_{2}{ }^{-}$ & -158.002992 \\
triangular $\mathrm{NaO}_{2}{ }^{-}$ & -312.808362 \\
linear $\mathrm{NaO}_{2}{ }^{-}$ & -312.761153 \\
bent $\mathrm{NaO}_{2}{ }^{-}$ & -312.808730 \\
$\mathrm{CO}_{3}{ }^{2-}$ & -263.813940 \\
$\mathrm{O}_{2}$ & -150.398101 \\
$\mathrm{CO}_{3}{ }^{-}$ & -264.004287 \\
$\left(\mathrm{CO}_{3}{ }^{2-}\right)_{2}$ & does not exist \\
$\mathrm{CO}_{5}{ }^{2-}$ & -414.253439 \\
$\mathrm{CO}_{2}$ & -188.740224 \\
$\mathrm{CO}_{4}{ }^{-}$ & -339.115387 \\
$\mathrm{O}_{2}{ }^{2-}$ & -150.099115 \\
$\mathrm{KCO}_{3}^{-}$ & -864.021719 \\
$\mathrm{LiCO}_{3}{ }^{-}$ & -271.636945 \\
$\mathrm{NaCO}_{3}^{-}$ & -426.380743 \\
$\mathrm{H}_{2} \mathrm{O}_{2}{ }^{-}$ & -151.679163 \\
$\mathrm{~K}^{+}$ & -599.835047 \\
$\mathrm{Li}^{+}$ & -7.349196 \\
$\mathrm{Na}^{+}$ & -162.158495 \\
triangular $\mathrm{KO}_{2}$ & -750.488221 \\
linear $\mathrm{KO}_{2}$ & -750.463320 \\
triangular $\mathrm{LiO}_{2}$ & -158.074822 \\
linear $\mathrm{LiO}_{2}$ & -158.041282 \\
triangular $\mathrm{NaO}_{2}$ & -312.833169
\end{tabular}

${ }^{a}$ Sum of electronic and thermal free energies; calculations done at 923 $\mathrm{K}$ and $1 \mathrm{~atm} .{ }^{b} 1 \mathrm{au}=627.5095 \mathrm{kcal} / \mathrm{mol}$.

also matched by the theoretical $\mathrm{O}-\mathrm{C}-\mathrm{O}$ bend and stretch vibration at $603 \mathrm{~cm}^{-1}$.

A modification of the above prediction (eq 2) is to include the reaction of $\mathrm{CO}_{4}{ }^{2-}$ with $\mathrm{K}^{+}$to form $\mathrm{KCO}_{4}^{-}$via eq 3:

$$
\mathrm{CO}_{4}^{2-}+\mathrm{K}^{+} \leftrightarrows \mathrm{KCO}_{4}^{-} \quad\left(\Delta G^{\circ}=-221.8 \mathrm{kcal}\right)
$$

The broad peak at $982 \mathrm{~cm}^{-1}$ can be represented by the $\mathrm{KCO}_{4}^{-}$bands (Table 1) from 888 to $1122 \mathrm{~cm}^{-1}$. The sum of these bands (weighted) is $964 \mathrm{~cm}^{-1}$. There is also a weaker vibration at $602 \mathrm{~cm}^{-1}$. It is likely that the observed spectrum is due to a combination of these vibrations for $\mathrm{CO}_{4}{ }^{2-}$ and $\mathrm{KCO}_{4}{ }^{-}$.

Presence of $\mathrm{O}_{2}^{-}$. Itoh ${ }^{3,5}$ combined electrochemical analysis with surface-enhanced Raman scattering (SERS) to study the $\mathrm{Li} / \mathrm{K}$ carbonate eutectic at $923 \mathrm{~K}$ and the $\mathrm{Li}_{2} \mathrm{CO}_{3}$ melt at $1123 \mathrm{~K}$. Raman lines were observed at 800, 1047, and $1080 \mathrm{~cm}^{-1}$ for both melts and at $735 \mathrm{~cm}^{-1}$ for the $\mathrm{Li}_{2} \mathrm{CO}_{3}$ melt in the presence of $\mathrm{O}_{2}$. Their results ${ }^{3,5}$ suggested that oxygen reduction in the $\mathrm{Li}_{2} \mathrm{CO}_{3}$ melt involved only peroxide ions while oxidation in the $\mathrm{Li} / \mathrm{K}$ carbonate eutectic involved the peroxide and superoxide species. The observed ${ }^{3,5}$ Raman lines at $800 \mathrm{~cm}^{-1}$ can be assigned to the $768 \mathrm{~cm}^{-1}$ Raman band for the triangular form of $\mathrm{LiO}_{2}$ given in Table 1. The linear (higher energy) form of $\mathrm{LiO}_{2}^{-}$also has a band at $772 \mathrm{~cm}^{-1}$ that cannot be completely ruled out. In the $\mathrm{Li}_{2} \mathrm{CO}_{3}$ melt, $\mathrm{LiO}_{2}{ }^{-}$has theoretical Raman lines at 949 and $1081 \mathrm{~cm}^{-1}$ (Table 1$)$. In the $\mathrm{Li} / \mathrm{K}$ eutectic melt, the same lines can be represented by those mentioned above for the $\mathrm{Li}_{2} \mathrm{CO}_{3}$ melt in addition to the 804 and $1080 \mathrm{~cm}^{-1}$ Raman bands calculated for the triangular and linear versions of $\mathrm{KO}_{2}{ }^{-}$.

Energetic Limitations on Raman Assignments. The data given in Table 2 indicate that certain of the theoretically possible forms are more favored than others. The three forms of $\mathrm{KO}_{2}^{-}$should exist (gas phase) in the approximate ratio of 85:12:1 for the bent, linear, and triangular forms. The assignment of the strong $\mathrm{KO}_{2}{ }^{-}$Raman band at $830 \mathrm{~cm}^{-1}$ with the theoretical vibration at $804 \mathrm{~cm}^{-1}$ is made on the basis of a theoretically strong vibration that matches the experimental result.

The ratio of the linear form of the triangular form of $\mathrm{LiO}_{2}{ }^{-}$to the linear form is approximately $1.7 \times 10^{8}: 1.00$. This effectively rules out any contribution from the triangular form of $\mathrm{LiO}_{2}^{-}$. The possible forms of $\mathrm{NaO}_{2}^{-}$theoretically exist in the ratio approximately $1.10: 1.00$ for the bent to triangular forms. The higher energy form (vs triangular and bent forms) of linear $\mathrm{NaO}_{2}{ }^{-}$formation limits its participation as a contributing species. The triangular forms of $\mathrm{KO}_{2}$ and $\mathrm{LiO}_{2}$ exist in ratios of approximately $720: 1$ and $10^{4}: 1$ to their linear forms, respectively. It is unlikely that the linear forms of $\mathrm{KO}_{2}$ and $\mathrm{LiO}_{2}$ contribute extensively to the Raman spectra of either species.

\section{CONCLUSIONS}

In general, the various predictions of high temperature species made in previous articles are reasonably accurate once a coordinating monovalent cation is attached to the anion under discussion.

\section{AUTHOR INFORMATION}

\section{Corresponding Author}

*E-mail: Bob.Carper@wichita.edu.

\section{Notes}

The authors declare no competing financial interest.

\section{ACKNOWLEDGMENTS}

The background for this work was developed during a Mercator Visiting Professorship (W.R.C.) at RWTH, Aachen, Germany. Partial support from the Air Force Office of Scientific Research to W.R.C. is also gratefully acknowledged.

\section{REFERENCES}

(1) Bates, J. B.; Brooker, M. H.; Quist, A. S.; Boyd, G. E. J. Phys. Chem. 1972, 76, 1565-1571.

(2) Chen, L.-J.; Chang, X.; Lin, C.-J.; Huang, C. M. Electrochim. Acta 2002, 47, 1475-1480.

(3) Itoh, T.; Abe, K.; Dokko, K.; Mohamedi, J.; Uchida, I.; Kasuya, A. J. Electrochem. Soc. 2004, 151, A2042-A2046.

(4) Chen, L.-J.; Lin, C.-J.; Zuo, J.; Song, L.-C.; Huang, C.-M. J. Phys. Chem. B 2004, 108, 7553-7556.

(5) Itoh, T.; Maeda, T.; Kasuya, A. Faraday Discuss. 2006, 132, 95109.

(6) Mizuhata, M.; Ohta, T.; Doki, S. Electrochemistry 2009, 77, 721724.

(7) McPhail, S. J. Adv. Sci. Technol. 2010, 72A, 283-290.

(8) Zaza, F.; Paoletti, C.; LoProst, R.; Simonetti, E.; Pasqual, M. Int. J. Hydrogen Energy 2011, 36, 8119-8125.

(9) Antolini, E. Appl. Energy 2011, 88, 4274-4293.

(10) Sanchez, D.; Munoz de Escalona, J. M.; Chacartegui, R.; Munoz, A.; Sanchez, T. J. Power Sources 2011, 196, 4347-4354. 
(11) Griffiths, T.; Vokovich, V. A. Nucl. Technol. 2008, 163, 382400.

(12) Griffiths, T.; Vokovich, V. A.; Carper, W. R. In Molten Salts and Ionic Liquids: Never the Twain?; Gaune-Escard, M., Seddon, K. R., Eds.; Wiley: Hoboken, NJ, 2010; pp 151-167.

(13) Griffiths, T.; Vokovich, V. A.; Carper, W. R. Struct. Chem. 2010, 21, 291-297.

(14) Talaty, E. A.; Raja, S.; Storhaug, V. J.; Dolle, S.; Carper, W. R. J. Phys. Chem. B 2004, 108, 13177-13184.

(15) Heimer, N. E.; Del Sesto, R. E.; Meng, Z.; Wilkes, J. S.; Carper, W. R. J. Mol. Liq. 2006, 124, 84-95.

(16) Carper, W. R.; Langenwalter, K.; Nooruddin, N. S.; Kullman, M. J.; Gerhard, D.; Wasserscheid, P. J. Phys. Chem. B 2009, 113, 20312041.

(17) Berg, R. W. Monatsh. Chem. 2007, 135, 1045-1075.

(18) Iwata, K.; Okajima, H.; Saha, S.; Hamaguchi, H. Acc. Chem. Res. 2007, 40, 1174-1181.

(19) Frisch, M. J.; Trucks, G. W.; Schlegel, H. B.; Scuseria, G. E.; Robb, M. A.; Cheeseman, J. R.; Montgomery, J. A., Jr.; Vreven, T.; Kudin, K. N.; Burant, J. C.; Millam, J. M.; Iyengar, S. S.; Tomasi, J.; Barone, V.; Mennucci, B.; Cossi, M.; Scalmani, G.; Rega, N.; Petersson, G. A.; Nakatsuji, H.; Hada, M.; Ehara, M.; Toyota, K.; Fukuda, R.; Hasegawa, J.; Ishida, M.; Nakajima, T.; Honda, Y.; Kitao, O.; Nakai, H.; Klene, M.; Li, X.; Knox, J. E.; Hratchian, H. P.; Cross, J. B.; Bakken, V.; Adamo, C.; Jaramillo, J.; Gomperts, R.; Stratmann, R. E.; Yazyev, O.; Austin, A. J.; Cammi, R.; Pomelli, C.; Ochterski, J. W.; Ayala, P. Y.; Morokuma, K.; Voth, G. A.; Salvador, P.; Dannenberg, J. J.; Zakrzewski, V. G.; Dapprich, S.; Daniels, A. D.; Strain, M. C.; Farkas, O.; Malick, D. K.; Rabuck, A. D.; Raghavachari, K.; Foresman, J. B.; Ortiz, J. V.; Cui, Q.; Baboul, A. G.; Clifford, S.; Cioslowski, J.; Stefanov, B. B.; Liu, G.; Liashenko, A.; Piskorz, P.; Komaromi, I.; Martin, R. L.; Fox, D. J.; Keith, T.; Al-Laham, M. A.; Peng, C. Y.; Nanayakkara, A.; Challacombe, M.; Gill, P. M. W.; Johnson, B.; Chen, W.; Wong, M. W.; Gonzalez, C.; Pople, J. A. Gaussian 03, revision D.02; Gaussian, Inc.: Wallingford, CT, 2004. 